\title{
Présentation du C.E.P.N.
}

\author{
H. P. JAMMET (*) \\ (mamuscrit reçu le 15 décembre 1980)
}

\begin{abstract}
RÉSUMÉ
Le C.E.P.N., Centre d'étude sur l'évaluation de la protection dans le domaine nucléaire est une association régie par la loi de 1901, créée par E.D.F. et le C.E.A. en mai 1976. Le Dr JAMMET qui fut l'un des promoteurs de cette création, retrace ici les circonstances et les motivations qui l'on déterminée et il présente de façon générale les objectifs et les programmes actuellement développés par ce centre.
\end{abstract}

ABSTRACT

The center for the assessment of protection in the nuclear field (C.E.P.N.) was set up by Électricité de France (E.D.F.) and the Commissariat à l'Énergie Atomique (C.E.A.) in May 1976. Dr JAMMET, one of its promoters, relates the circumstances and motivations of its creation and presents the general lines of the purposes and programmes presently developed by the center.

Question : Quelles ont été les motivations de la création du C.E.P.N.?

Dr Jammet : Dans le domaine radiologique, les bases scientifiques et techniques servant à la réglementation sont probablement beaucoup mieux connues que dans le cas de la plupart des autres nuisances industrielles que nous connaissons. Les données sur lesquelles reposent les choix de radioprotection sont très diverses et sont issues de secteurs scientifiques extrêmement variés allant des disciplines fondamentales de la physique et de la biologie jusqu'aux sciences de l'ingénieur. Avec le développement de ces multiples travaux, des approches de type synthétique et transversal sont apparues peu à peu nécessaires, si l'on veut que s'instaure un certain dialogue social entre les responsables et les personnes exposées, qu'il s'agisse du public ou des travailleurs. En effet, devant la variété, la complexité et le caractère extrêmement analytique des données mises en jeu, il est nécessaire pour comprendre véritablement, pour pénétrer les fondements scientifiques et techniques dans ce domaine, de faire un effort de synthèse très difficile pour un non-spécialiste. Ainsi,la nécessité de développer des études permettant de mettre en perspective les différentes dimensions qui interviennent dans les choix de protection est-elle apparue peu à peu.

(*) Commissariat à l'Énergie Atomique, Institut de Protection et de Sûreté Nucléaire, Département de Protection, B.P. ${ }^{\circ} 6,92260$ Fontenay-aux-Roses.

RADIOPROTECTION, VOL. $16-0033-8451 / 1981 / 125 / \$ 5.00 /$ C B Bordas-Dunod. 
Par ailleurs, avec le développement des applications industrielles de la radioactivité et des programmes électronucléaires, les choix en matière de radioprotection ont pris une dimension économique et sociale qui apparaît de plus en plus fondamentale. Aussi, tous les radioprotectionnistes seront-ils d'accord pour reconnaître que ces différentes dimensions ne doivent pas être seulement saisies de façon purement empirique, mais doivent aussi donner lieu à des études présentant toutes les garanties d'objectivité et de rigueur. Ces préoccupations se reflètent dans les travaux des différentes organisations internationales qui ont vu le jour au cours des dernières décennies. La prise en compte des dimensions économiques et sociales est apparue dans les recommandations formulées par la Commission Internationale de Protection Radiologique, dès les années 1950, mais il aura fallu attendre plus de vingt ans pour que cette idée générale, pourtant simple, se matérialise dans des recommandations plus concrètes et aboutisse à des travaux qui intègrent, de façon explicite, à la fois la mesure des coûts entraînés par les choix de protection et les dimensions psycho-sociologiques de ces choix. Je dois dire que ces travaux, s'ils sont récents à propos du risque radiologique, s'inscrivent dans un développement plus général qui est celui qu'ont connu des disciplines nouvelles, en développement, comme l'économie de l'environnement et la socio-économie de la santé. Ces travaux concernent, de façon générale, la description et l'explication des comportements et des décisions en matière de protection de l'environnement et d'organisation de la santé et de la médecine préventive. C'est donc dans cette perspective que l'on doit comprendre l'intérêt de développer des études servant à la fois à justifier nos pratiques et aussi à enrichir notre perspective de la radioprotection.

\section{Question : Pouvez-vous nous préciser les objectifs du C.E.P.N.?}

Dr JAMMET : Le C.E.P.N. est un organisme d'étude et de recherche qui n'a pas de responsabilité opérationnelle. Ses objectifs sont d'ordre méthodologique; il s'agit de développer les méthodes qui permettent d'intégrer les dimensions économiques et sociales dans les choix de protection radiologique. Je voudrais insister pour dire qu'il s'agit de recherche méthodologique. Ces méthodes ne sont pas parfaitement connues et il s'agit de mettre au point des outils qui soient adaptés aux problèmes que nous rencontrons dans le domaine de la radioprotection. Pour faire de la méthodologie il faut connaître, au préalable, de façon très profonde et intime, les multiples données biologiques et techniques qui constituent les éléments d'information de base indispensables. Il y a donc une tâche très importante de collecte et d'élaboration de données. C'est pourquoi le C.E.P.N., depuis sa création, a commencé à développer des études de cas en relation avec certains utilisateurs et praticiens de la radioprotection. Un autre aspect des études du C.E.P.N. concerne les approches comparatives. Il paraît indispensable, lorsque l'on veut analyser le cas du risque radiologique, de le replacer dans le contexte plus général des décisions concernant les risques liés à d'autres secteurs industriels. Aussi, parmi les activités et les objectifs du C.E.P.N., une part importante est réservée aux analyses comparatives: comparaison entre différentes industries en terme de risque, comparaison des 
risques liés à différentes technologies de production d'électricité mais aussi comparaison des expositions radiologiques d'origine industrielle certes, mais aussi d'origine médicale (radiodiagnostic) ou naturelle. Enfın, dernier aspect, et qui est loin d'être négligeable, les perceptions sociales des risques constituent désormais un aspect important qui doit être pris en compte par les responsables; par conséquent, une étude systématique des dimensions subjectives et psychologiques de la perception du risque doit également être abordée avec des méthodes appropriées. Ce dernier aspect rentre également dans les objectifs de recherche et d'étude de ce centre.

Question : Quels sont les moyens actuels dont dispose le C.E.P.N. pour effectuer ces travaux?

Dr JAMMET : Le C.E.P.N. dispose actuellement d'une douzaine d'ingénieurs et de chercheurs et d'un personnel administratif. Ces ingénieurs et chercheurs ont des statuts variés, certains d'entre eux ont été mis à disposition par les organismes fondateurs, C.E.A. et E.D.F., d'autres viennent d'organismes publics de recherche comme l'I.N.S.E.R.M. $\left({ }^{1}\right)$ et le C.N.R.S., d'autres enfin sont salariés directement par le C.E.P.N. En ce qui concerne les sources de financement, elles sont essentiellement de deux origines: d'une part, les ressources propres de l'organisme qui lui sont versées annuellement sous forme d'une cotisation par chacun des membres de l'association et qui lui permettent d'assurer un ensemble de travaux qui lui sont demandés par les autorités qui contrôlent cet organisme. D'autre part, le C.E.P.N., en tant qu'association, peut réaliser des travaux sur contrat avec tout organisme qui lui en fait la demande et sur les sujets qui correspondent à ses objectifs. Depuis que le C.E.P.N. fonctionne, c'est-à-dire depuis 4 ans, on peut considérer que ces deux sources de financement ont contribué à peu près à part égale aux ressources. La plupart des contrats demandés au C.E.P.N., jusqu'à présent, proviennent des Communautés Européennes et des organismes fondateurs, c'est-à-dire le C.E.A. et l'E.D.F. Cette formule a le mérite, au-delà des ressources supplémentaires qui sont ainsi apportées, de mettre en contact direct les ingénieurs du C.E.P.N. avec des interlocuteurs qui sont les utilisateurs des résultats et sont donc en situation de proposer des sujets d'études et de recherches concrets.

Sur le plan de l'organisation pratique, cet organisme est situé dans les locaux du Département de protection du C.E.A. à Fontenay-aux-Roses, donc au sein de l'Institut de Protection et de Sûreté Nucléaire. Le C.E.P.N. est dirigé sur le plan scientifique par une personne extérieure aux deux institutions fondatrices, M. FAGNANI, chercheur à l'I.N.S.E.R.M., qui a une expérience ancienne dans le domaine de l'économie de la santé et de la gestion des programmes de santé et de prévention, ce qui permet d'assurer une liaison avec un ensemble d'activités de recherche dans le domaine biomédical et dans celui de l'économie de la santé et de l'environnement. Par ailleurs, M. FAGNANı assure également, en parallèle, la

( ${ }^{1}$ ) Institut national de la santé et de la recherche médicale. 
direction d'une Formation de recherche associée de l'I.N.S.E.R.M. sur le thème plus général de l'évaluation des risques et des actions de prévention. Cette Formation de recherche associée est située dans les locaux du C.E.P.N. et regroupe, pour l'instant, deux chercheurs I.N.S.E.R.M. et ouvre la possibilité, à terme, au développement de ses activités en liaison avec celles du C.E.P.N. Ainsi, nous espèrons avoir pu mettre en place une organisation qui permette, à la fois, les liaisons avec l'ensemble des activités de recherche médicale, avec les utilisateurs de l'industrie et du C.E.A. qui sont directement concernés par les problèmes de risques radiologiques, et ceci dans les meilleures conditions possibles.

Sur le plan purement administratif, le C.E.P.N., en tant qu'association, Loi de 1901, est géré conformément aux règles juridiques générales qui régissent ce type d'association. Une assemblée générale a été constituée avec une représentation à part égale des deux organismes fondateurs, le C.E.A. et l'E.D.F. Un conseil d'administration est issu des membres de cette assemblée générale; ce conseil, qui se réunit plusieurs fois par an, défınit les objectifs des études et des recherches à entreprendre. Par ailleurs, un comité scientifique qui rassemble des personnalités scientifiques d'institutions diverses et des responsables intéressés par les études se réunit régulièrement pour donner des conseils et des orientations sur le travail effectué. Le directeur scientifique du C.E.P.N. rend compte de ses activités devant ces différentes assemblées.

Question : Comment sont diffusés les résultats des travaux effectués? Sontils destinés au public ou sont-ils réservés à un usage interne?

Dr JAMMET : Comme je vous l'ai expliqué, le premier critère qui doit caractériser les études et recherches effectuées est leur caractère scientifique. Par conséquent, les travaux effectués par le C.E.P.N. doivent être considérés comme devant subir le même type de diffusion et les mêmes jugements de qualité que ceux que l'on considère, en général, pour la production scientifique. Autrement dit, les travaux du C.E.P.N. subissent une diffusion à travers les publications scientifiques telles qu'elles existent actuellement, c'est-à-dire les revues spécialisées, les colloques, les réunions scientifıques organisées à différentes occasions. Par ailleurs, comme je vous l'ai dit tout à l'heure, une part importante des travaux sont effectués sur contrat et donnent lieu à un ensemble de rapports qui sont utilisés directement par les contractants. La diffusion de l'ensemble des travaux effectués s'opère sous le contrôle des membres du conseil d'administration. Ces travaux, qui intègrent les disciplines économiques et les sciences humaines, peuvent surprendre certains d'entres nous. Mais je crois qu'il faut faire l'effort d'accueillir cette nouvelle forme de recherche dans notre domaine au même titre que les travaux traditionnels, à caractère plus technique.

Question: Sur quels thèmes plus spécifiques ont porté les travaux effectués par le C.E.P.N. depuis sa création?

Dr JAMmET : Une première catégorie concerne l'optimisation des choix de radioprotection dans le cadre des installations du cycle du combustible nucléaire. L'un des premiers problèmes qui se pose pour aborder les questions 
sous cet angle concerne la disponibilité d'une information suffisante sur les plans technique et sanitaire aussi bien qu'économique. Par conséquent, une part importante des activités du C.E.P.N. a été consacrée, jusqu'à présent, à un recueil de données, françaises de préférence, pouvant faire l'objet d'une évaluation critique et possédant donc un certain degré de frabilité et de crédibilité. Ainsi, un travail de longue haleine en liaison avec le S.E.P.T.E.N. $\left({ }^{2}\right)$ à l'E.D.F. a été consacré à l'analyse des systèmes de traitement des effluents dans les centrales nucléaires françaises. Sur la base des données recueillies, un travail méthodologique approfondi a porté sur les méthodes d'aide à la décision susceptibles d'être utilisées dans le cadre de l'optimisation de la radioprotection, qu'il s'agisse des méthodes classiques comme l'analyse coût-bénéfice, ou de celles moins connues chez les radioprotectionnistes comme les approches de type multicritère.

Actuellement, les recherches s'orientent davantage vers la radioprotection des travailleurs et une première étude est en cours, en liaison avec le Département de radioprotection d'E.D.F., concernant l'évaluation économique des mesures de protection dans les centrales. Il s'agit là d'une question qui suscite l'intérêt de l'exploitant tant au niveau de la conception qu'à celui de l'exploitation de ses centrales.

Le C.E.P.N. s'est également penché sur le problème de l'optimisation de la protection des travailleurs dans les mines d'uranium. C'est, comme vous le savez, l'un des points du cycle qui mérite un intérêt particulier. Enfın, une série de travaux a été consacrée à l'évaluation des risques liés au transport des matières radioactives. Ces études ne se sont pas limitées à la stricte évaluation des probabilités d'accidents et de leurs conséquences dans le cas de la France, mais ont également intégré les dimensions économiques de la protection dans les transports, illustrant ainsi l'application possible du principe d'optimisation dans le domaine de la sûreté. La méthodologie et la base de données développées à l'occasion de ces travaux permettent d'envisager, à présent, la comparaison avec les risques liés aux transports d'autres matières dangereuses.

La comparaison des risques et de leur mode de gestion constitue, en effet, la deuxième grande catégorie de travaux du C.E.P.N. A ce sujet, je dois d'abord mentionner l'étude soutenue financièrement par les Études et Recherches d'E.D.F. concernant la comparaison des risques des différentes filières de production d'électricité, qui est probablement la seule étude de ce type effectuée dans le cadre européen. Le C.E.P.N. a réalisé, depuis 3 ans, un travail de synthèse des données concernant les risques comparés des énergies classiques de production d'électricité - fuel, charbon, nucléaire - et des énergies nouvelles basées sur le solaire. Ce travail a fait l'objet d'un ensemble de présentations à différentes occasions et constitue l'une des rares études fondées sur des scénarios qui soient adaptés à la situation spécifique de la France. Par ailleurs, d'autres études ont été effectuées, visant non pas à comparer des niveaux de risque mais plutôt des modes de gestion. Ces études ont porté sur la comparaison des efforts consentis dans le cas du risque radiologique à ceux relatifs à d'autres nuisances.

(2) Service d'études et projets thermiques et nucléaires.

VOL. $16-\mathrm{N}^{\circ} 2$ 
Le problème est d'évaluer dans quelle mesure il y a cohérence entre les exigences que l'on a dans les divers secteurs industriels. Ainsi le C.E.P.N. développe-t-il actuellement des études visant à comparer les dépenses de protection dans les installations nucléaires à celles des centrales thermiques classiques pour réduire les pollutions atmosphériques ou bien encore, à celles de l'industrie de l'amiante pour réduire les risques pour les travailleurs. Bien que, comme je l'ai souligné tout à l'heure, les recherches du C.E.P.N. aient essentiellement un caractère méthodologique, vous voyez, à travers ces exemples, que l'un des soucis primordiaux dans l'orientation des travaux, a été de mener la réflexion théorique à partir de cas concrets. C'est en effet sur la base de données suffisamment fiables et riches qu'il convient de mettre au point les outils d'aide à la décision qui permettent d'introduire les dimensions économiques et sociales dans le cadre de la gestion du risque radiologique.

Radioprotection : Nous vous remercions d'avoir bien voulu éclairer nos lecteurs sur les objectifs du C.E.P.N. dont les travaux devraient contribuer à dépassionner un peu le débat qui s'est institué vis-à-vis des risques des rayonnements ionisants. 